\title{
ON THE NUMBER OF NON-ZERO DIGITS OF INTEGERS IN MULTI-BASE REPRESENTATIONS
}

\author{
CS. BERTÓK, L. HAJDU, F. LUCA, AND D. SHARMA \\ Dedicated to A. Sárközy on the occasion of his 75th birthday
}

\begin{abstract}
We prove various finiteness theorems for integers having only few non-zero digits in different multi-base representations simultaneously.
\end{abstract}

\section{INTRODUCTION}

It is an old problem to study integers having only a 'few' non-zero digits in some classical base $b$ representation, see e.g. papers by Erdős, Mauduit, Pomerance, Sárközy [6, 7, 15, 16, 17] and the references there. On the other hand, if a number $n$ has to hold certain other arithmetical property, it may happen that it must have 'many' digits. This is the case when $n$ belongs to some recurrence sequence; see e.g. Bugeaud, Cipu and Mignotte [4], Luca [13] and Stewart [21] for effective results in this direction.

The number of non-zero digits of integers, and integers with fixed number of non-zero digits is also investigated with respect to other types of bases, e.g. with respect to linear recurrence number systems, cf. $[19,22]$. Another generalization of the classical number systems is given by the so-called multi-base representations, when instead of linear combinations of powers of a fixed number $b$, one can combine products of powers of fixed primes. For related problems and results see e.g. the papers $[1,2,5,10,11,12,18]$ and the references therein.

It is an interesting question to study integers having only 'few' nonzero digits in different bases simultaneously. Here we mention two results. Senge and Straus [20] proved that the number of those integers, whose number of non-zero digits in two different bases $b_{1}$ and $b_{2}$ with $\log b_{1} / \log b_{2} \notin \mathbb{Q}$ remains under some fixed bound, is finite. Later, Stewart [21] gave a more precise, effective version of this result.

2010 Mathematics Subject Classification. 11A63, 11A67, 11D61.

Key words and phrases. Multi-base representation, number of non-zero digits, $S$-unit equations.

Research supported in part by the OTKA grants K100339 and K115479. 
In this paper, as a generalization of the problem mentioned in the previous paragraph, we study integers having only 'few' non-zero digits in different multi-base representations simultaneously. To set the problem precisely, we need to introduce some notation.

Let $S$ be a finite set of primes, and write $\mathbb{Z}_{S}$ (resp. $\mathbb{Z}_{S}^{+}$) for the set of integers (resp. positive integers) having no prime divisors outside $S$. A multi-base representation of an integer $n$ is an expression of the form

$$
n=u_{1}+\cdots+u_{t}
$$

with $u_{1}, \ldots, u_{t} \in \mathbb{Z}_{S}$. If $S=\{p\}$ and we require that $u_{1}, \ldots, u_{t} \in \mathbb{Z}_{S}^{+}$, we get several expansions of $n$ as sums of powers of $p$, with the shortest one (namely, the one with fewest terms) being the usual expansion of $n$ in base $p$. For an integer $n$, we write $w_{S}(n)$ for the minimal $t$ for which (1) holds with some $u_{1}, \ldots, u_{t} \in \mathbb{Z}_{S}$. If $n>0$ and we also require that $u_{1}, \ldots, u_{t} \in \mathbb{Z}_{S}^{+}$, we then write $w_{S}^{+}(n)$ instead.

In what follows, we prove various finiteness theorems for integers $n$ with 'small' values of $w_{S}^{+}(n)$ with respect to different sets $S$ simultaneously. To prove our results, we use Baker's method for linear forms in logarithms, a deep theorem of Evertse [8] bounding the number of nondegenerate solutions of $S$-unit equations and a local method of Bertók and Hajdu [3] developed for the resolution of exponential equations over $\mathbb{Z}$.

\section{NeW RESUlts}

Our first theorem concerns the general case.

Theorem 2.1. Let $k$ be a positive integer, $S_{1}, \ldots, S_{k}$ be finite sets of primes such that $S_{1} \cap \cdots \cap S_{k}=\emptyset$. Then for any $T$ the inequality

$$
w_{S_{1}}^{+}(n)+\cdots+w_{S_{k}}^{+}(n) \leq T
$$

is valid only for finitely many integers $n$. Further, the number of such integers $n$ is at most $c_{1}=c_{1}(T, k, s)$, where $c_{1}$ is an effectively computable constant depending only on $T, k$ and $s:=\left|S_{1} \cup \cdots \cup S_{k}\right|$.

Remark 1. Note that the condition $S_{1} \cap \cdots \cap S_{k}=\emptyset$ in the above theorem is necessary. Indeed, if $p \in S_{1} \cap \cdots \cap S_{k}$ would hold with some prime $p$, then for $T:=k \geq 1$ we would have

$$
w_{S_{1}}^{+}(n)+\cdots+w_{S_{k}}^{+}(n) \leq T
$$

for all $n=p^{\alpha}(\alpha \geq 0)$.

Our second result gives an effective bound in a special case. 
Theorem 2.2. Let $\ell$ be a positive integer, $S_{1}=\left\{p_{1}, \ldots, p_{\ell}\right\}$ and $S_{2}=\{q\}$, where $p_{1}, \ldots, p_{\ell}, q$ are distinct primes. If $n$ is a positive integer with $n>e^{e^{e}}$ such that $w_{S_{1}}^{+}(n)=1$, then we have

$$
w_{S_{2}}^{+}(n)>\frac{c_{2} \log \log n}{\log \log \log n},
$$

where $c_{2}=c_{2}\left(\ell, p_{1}, \ldots, p_{\ell}, q\right)$ is an effectively computable positive constant depending only on $\ell, p_{1}, \ldots, p_{\ell}, q$.

Remark 2. The condition $q \notin S_{1}$ is necessary. This can be easily checked by a similar example as in Remark 1 . Further, we note that if $S_{i}$ are not sets of primes, but sets of multiplicatively independent integers instead, then after the necessary modifications our theorems still hold.

Finally, we give a complete list of integers $n$ having only a 'few' nonzero digits for some fixed choices of sets $S_{1}, S_{2}$. Note that in the cases considered, though the number of solutions can be bounded e.g. by results of Evertse [8], there are no tools available which would effectively bound the solutions themselves. To find the solutions explicitly, we apply a method of Bertók and Hajdu [3].

Theorem 2.3. Let $S_{1}, S_{2}$ be disjoint non-empty sets of primes with $S_{1} \cup S_{2}=\{2,3,5\}$. Then

$$
w_{S_{1}}^{+}(n)+w_{S_{2}}^{+}(n) \leq 4
$$

implies that if

(1) $S_{1}=\{2\}$ and $S_{2}=\{3,5\}$ then $n \in\{1,2,3,4,5,6,8,9,10,12$, $16,18,20,24,25,32,34,36,40,48,72,80,81,96,128,130,136$, $144,160,258,260,288,384,640,1152,2050,2052,4104,32832\}$;

(2) $S_{1}=\{3\}$ and $S_{2}=\{2,5\}$ then $n \in\{1,2,3,4,5,6,9,10,12,18$, $27,28,30,36,54,81,82,84,90,108,162,252,270,324,729,756$, $810,6561,6570\}$

(3) $S_{1}=\{5\}$ and $S_{2}=\{2,3\}$ then $n \in\{1,2,3,5,6,10,25,26,27$, $30,50,125,126,130,150,625,630,650,3125,3126,15625,78750\}$.

\section{Proof of Theorem 2.1}

To prove Theorem 2.1, we need to introduce some notions and notation.

Let $a_{1}, \ldots, a_{\ell} \in \mathbb{Q}^{*}$. Consider the equation

$$
a_{1} x_{1}+\cdots+a_{\ell} x_{\ell}=0
$$


in $x_{1}, \ldots, x_{\ell} \in \mathbb{Z}_{S}$. A solution $\left(x_{1}, \ldots, x_{\ell}\right)$ of the above equation is said to be non-degenerate if

$$
\sum_{i \in I} a_{i} x_{i} \neq 0 \text { for each non-empty } I \subset\{1, \ldots, \ell\}
$$

and degenerate otherwise. Further, two solutions $\left(x_{1}, \ldots, x_{\ell}\right)$ and $\left(y_{1}, \ldots, y_{\ell}\right)$ of (2) are called proportional if for some $z \in \mathbb{Q}^{*}$, we have

$$
x_{i}=z y_{i} \text { for } i=1, \ldots, \ell .
$$

Lemma 3.1. Let $s=|S|$. Then equation (2) has at most

$$
\left(2^{35}(\ell-1)^{2}\right)^{(\ell-1)^{3} s}
$$

non-degenerate solutions $\left(x_{1}, \ldots, x_{\ell}\right) \in \mathbb{Z}_{S} \times \cdots \times \mathbb{Z}_{S}$, no two of which are proportional.

Proof. The statement is a simple consequence of [8, Theorem 3].

Theorem 2.1 can be immediately deduced from the following result.

Proposition 3.1. Let $k \geq 2$ and let $t_{1}, \ldots, t_{k} \in \mathbb{N}$. For $i=1, \ldots, k$, let

$$
A_{i}=\left\{a_{i, 1}, \ldots, a_{i, t_{i}}\right\}
$$

be a set of $t_{i}$ positive integers. Then the number of positive integers $n$ such that for each $i$, there exist $u_{i, 1}, \ldots, u_{i, t_{i}} \in \mathbb{Z}_{S_{i}}^{+}$such that

$$
n=a_{i, 1} u_{i, 1}+\cdots+a_{i, t_{i}} u_{i, t_{i}},
$$

is at most

$$
\left(2^{35}(t-1)^{2}\right)^{(k-1)(t-1)^{4} s}
$$

where $t=t_{1}+\cdots+t_{k}$ and $s=\left|S_{1} \cup S_{2} \cup \cdots \cup S_{k}\right|$.

Proof. We prove the proposition by induction on $k$.

Let $S=S_{1} \cup S_{2} \cup \cdots \cup S_{k}$. Suppose that $k=2$. We will prove that the result holds in this case using induction on $t=t_{1}+t_{2}$. Suppose that $t=2$. Then $t_{1}=t_{2}=1$. We now show that the equation

$$
a_{1,1} u_{1,1}=a_{2,1} u_{2,1}
$$

in $\left(u_{1,1}, u_{2,1}\right) \in \mathbb{Z}_{S_{1}}^{+} \times \mathbb{Z}_{S_{2}}^{+}$has at most one solution. Indeed, the equation (3) implies that

$$
\frac{u_{1,1}}{u_{2,1}}=\frac{a_{2,1}}{a_{1,1}} .
$$

The claim follows by the coprimality of $u_{1,1}$ and $u_{2,1}$. Therefore, the result holds when $k=t=2$. Let $t \geq 3$ and assume that the result holds whenever $t_{1}+t_{2} \leq t-1$. We now consider the case $t_{1}+t_{2}=t$. We have to count the number of solutions of the $S$-unit equation

$$
a_{1,1} u_{1,1}+\cdots+a_{1, t_{1}} u_{1, t_{1}}=a_{2,1} u_{2,1}+\cdots+a_{2, t_{2}} u_{2, t_{2}},
$$


where $u_{1, j} \in \mathbb{Z}_{S_{1}}^{+}$and $u_{2, j} \in \mathbb{Z}_{S_{2}}^{+}$. By Lemma 3.1, this equation has at most

$$
\left(2^{35}(t-1)^{2}\right)^{(t-1)^{3} s}
$$

non-degenerate solutions. Next, we count the number of degenerate solutions. (Observe that if $t=3$, then $\left(t_{1}, t_{2}\right)=(1,2)$ or $(2,1)$ and hence all the solutions are non-degenerate. Therefore, while counting degenerate solutions, it is understood that $t \geq 4$.) For a degenerate solution, there exists a non-empty subset $I$ of $\left\{1, \ldots, t_{1}\right\}$ and a nonempty subset $J$ of $\left\{1, \ldots, t_{2}\right\}$ such that

$$
\sum_{i \in I} a_{1, i} u_{1, i}-\sum_{j \in J} a_{2, j} u_{2, j}=0
$$

but no proper subsum in this equation vanishes. Fix $I, J$. We count the number of solutions of (4) satisfying (5). Since $|I|+|J| \leq t-2$, it follows from Lemma 3.1 that the $S$-unit equation (5) has at most

$$
\left(2^{35}(t-3)^{2}\right)^{(t-3)^{3} s}
$$

non-degenerate solutions. Further, by the induction hypothesis, the equation

$$
\sum_{i \notin I} a_{1, i} u_{1, i}-\sum_{j \notin J} a_{2, j} u_{2, j}=0
$$

has at most

$$
\left(2^{35}(t-3)^{2}\right)^{(t-3)^{4} s}
$$

solutions. Hence, given $I, J$, we obtain that there are at most

$$
\left(2^{35}(t-3)^{2}\right)^{s(t-3)^{3}(t-2)}
$$

solutions. Varying $I$ and $J$, we obtain that the total number of degenerate solutions is at most

$$
2^{t}\left(2^{35}(t-3)^{2}\right)^{s(t-3)^{3}(t-2)} \leq \frac{1}{2}\left(2^{35}(t-1)^{2}\right)^{(t-1)^{4} s} .
$$

Thus, (4) has at most

$$
\left(2^{35}(t-1)^{2}\right)^{(t-1)^{3} s}+\frac{1}{2}\left(2^{35}(t-1)^{2}\right)^{(t-1)^{4} s} \leq\left(2^{35}(t-1)^{2}\right)^{(t-1)^{4} s}
$$

solutions. This completes the induction on $t$. Hence, the result holds for $k=2$.

Now let $k \geq 3$. Suppose that the result holds for every $k^{\prime}$ with $2 \leq k^{\prime}<k$. That is, given $k^{\prime}$ in the above range, we assume that the result is valid for all $t$ and for all choices of the sets $A_{i}$ and $S_{i}$. Note 
that $t=t_{1}+\cdots+t_{k} \geq k \geq 3$. We have to bound the number of solutions of the following system of $S$-unit equations:

$$
\begin{aligned}
& a_{1,1} u_{1,1}+\cdots+a_{1, t_{1}} u_{1, t_{1}}= a_{2,1} u_{2,1}+\cdots+a_{2, t_{2}} u_{2, t_{2}} \\
& \vdots \\
&=a_{k, 1} u_{k, 1}+\cdots+a_{k, t_{k}} u_{k, t_{k}},
\end{aligned}
$$

where $u_{i, j} \in \mathbb{Z}_{S_{i}}^{+}$. We mention that similar systems of $S$-unit equations have been studied by Evertse and Győry [9]. However, their theorems cannot be used directly here, so we apply some other results. Namely, by Lemma 3.1, the first equation in (6) has at most

$$
\left(2^{35}(t-2)^{2}\right)^{(t-2)^{3} s}
$$

non-degenerate solutions. For a degenerate solution, there exists a positive integer $l \leq t-2$ and distinct non-empty subsets $I_{1}, \ldots, I_{l} \subseteq$ $\left\{1, \ldots, t_{1}\right\}, J_{1}, \ldots, J_{l} \subseteq\left\{1, \ldots, t_{2}\right\}$ such that for $m=1, \ldots, l$,

$$
\sum_{i \in I_{m}} a_{1, i} u_{1, i}=\sum_{i \in J_{m}} a_{2, i} u_{2, i},
$$

but no proper subsum vanishes. Fix $I_{1}, \ldots, I_{l}, J_{1}, \ldots, J_{l}$. We count the number of solutions of system (6) satisfying the additional equations (8). By Lemma 3.1, for each $m=1, \ldots, l,(8)$ has, up to a factor of proportionality, at most

$$
\left(2^{35}(t-3)^{2}\right)^{(t-3)^{3} s}
$$

non-degenerate solutions. Let $\left(\left(u_{1, i}\right)_{i \in I_{m}},\left(u_{2, i}\right)_{i \in J_{m}}\right)$ be a solution of (8) with $\operatorname{gcd}\left(\left(u_{1, i}\right)_{i \in I_{m}},\left(u_{2, i}\right)_{i \in J_{m}}\right)=1$. Set

$$
a_{m}^{\prime}=\sum_{i \in I_{m}} a_{1, i} u_{1, i}\left(=\sum_{i \in J_{m}} a_{2, i} u_{2, i}\right)
$$

Then

$$
\left\{\left(\left(U_{m} u_{1, i}\right)_{i \in I_{m}},\left(U_{m} u_{2, i}\right)_{i \in J_{m}}\right): U_{m} \in \mathbb{Z}_{S_{1} \cap S_{2}}^{+}\right\}
$$

is precisely the set of solutions of (8) which are proportional to $\left(\left(u_{1, i}\right)_{i \in I_{m}},\left(u_{2, i}\right)_{i \in J_{m}}\right)$. The problem is thus reduced to considering the following system of equations in the variables $U_{1}, \ldots, U_{l}, u_{3,1} \ldots, u_{k, t_{k}}$ :

$$
\begin{gathered}
a_{1}^{\prime} U_{1}+\cdots+a_{l}^{\prime} U_{l}=a_{3,1} u_{3,1}+\cdots+a_{3, t_{3}} u_{3, t_{3}} \\
\vdots \\
=a_{k, 1} u_{k, 1}+\cdots+a_{k, t_{k}} u_{k, t_{k}} .
\end{gathered}
$$


Since $\left(S_{1} \cap S_{2}\right) \cap S_{3} \cap \cdots \cap S_{k}=\emptyset$, we apply the induction hypothesis for $k^{\prime}=k-1$ to conclude that the above system of equations has at most

$$
\left(2^{35}(t-2)^{2}\right)^{(k-2)(t-2)^{4} s}
$$

solutions. Hence, given $I_{1}, \ldots, I_{l}, J_{1}, \ldots, J_{l}$, we get at most

$$
\left(2^{35}(t-3)^{2}\right)^{(t-3)^{3} s(t-2)} \cdot\left(2^{35}(t-2)^{2}\right)^{(k-2)(t-2)^{4} s}
$$

solutions. Therefore the number of degenerate solutions is bounded by

$$
t^{t}\left(2^{35}(t-2)^{2}\right)^{(k-1)(t-2)^{4} s} \leq \frac{1}{2}\left(2^{35}(t-1)^{2}\right)^{(k-1)(t-1)^{4} s} .
$$

Combining the above bound (9) with (7), we obtain that the total number of solutions is at most

$$
\left(2^{35}(t-1)^{2}\right)^{(k-1)(t-1)^{4} s}
$$

This completes the induction and the proof of the proposition.

Proof of Theorem 2.1. Taking $t_{i}=w_{S_{i}}^{+}(n)$ and $A_{i}=\{1\}$ for all $i=$ $1, \ldots, k$ in Proposition 3.1, the statement immediately follows.

\section{Proof of Theorem 2.2}

To prove Theorem 2.2, we use a Baker type estimate due to Matveev [14]. For its formulation we need to introduce some notation.

For an algebraic number $\alpha$ of degree $D$ over $\mathbb{Q}$, the absolute logarithmic height of $\alpha$ is defined by

$$
h(\alpha)=\frac{1}{D}\left(\log a_{0}+\sum_{i=1}^{D} \log \max \left(1,\left|\alpha^{(i)}\right|\right)\right),
$$

where $a_{0}>0$ is the leading coefficient of the minimal polynomial of $\alpha$ over $\mathbb{Z}$ and the $\alpha^{(i)}$ 's are the conjugates of $\alpha$. Note that in the special case when $\alpha=p / q$ is a non-zero rational number with $\operatorname{gcd}(p, q)=1$, then $h(\alpha)=h(1 / \alpha)=\log \max \{|p|,|q|\}$.

The following result is due to Matveev [14].

Lemma 4.1. Assume that $\alpha_{1}, \ldots, \alpha_{r}$ are positive real algebraic numbers in a real algebraic number field of degree $D, d_{1}, \ldots, d_{r}$ are rational integers, and

$$
\Lambda:=\alpha_{1}^{d_{1}} \ldots \alpha_{r}^{d_{r}}-1
$$

is not zero. Set

$$
B \geq \max \left\{\left|d_{1}\right|, \ldots,\left|d_{r}\right|\right\},
$$

and

$$
A_{i} \geq \max \left\{D h\left(\alpha_{i}\right),\left|\log \alpha_{i}\right|, 0.16\right\}, \text { for all } i=1, \ldots, r \text {. }
$$


Then we have

$$
|\Lambda|>\exp \left(-1.4 \cdot 30^{r+3} r^{4.5} D^{2}(1+\log D)(1+\log B) A_{1} \cdots A_{r}\right) .
$$

Now we are ready to give the proof of Theorem 2.2.

Proof of Theorem 2.2. We combine arguments of Luca [13] and Stewart [21] with some other considerations.

Let $n$ be a positive integer with $w_{S_{1}}^{+}(n)=1$ and $w_{S_{2}}^{+}(n)=t$, and write

$$
u_{1}=n=v_{1}+\cdots+v_{t}
$$

with $u_{1} \in \mathbb{Z}_{S_{1}}^{+}$and $v_{1}, \ldots, v_{t} \in \mathbb{Z}_{S_{2}}^{+}$. Without loss of generality we may assume that $v_{1} \geq \cdots \geq v_{t}$.

We write

$$
u_{1}=p_{1}^{\alpha_{1}} \ldots p_{\ell}^{\alpha_{\ell}}, \quad v_{i}=q^{\beta_{i}} \quad(i=1, \ldots, t) .
$$

Let $B$ be the maximum of the exponents appearing in (12).

Equation (11) can be rewritten as

$$
u_{1}-v_{1}=v_{2}+\cdots+v_{t} .
$$

Since $u_{1} \neq v_{1}$ and $v_{1} \neq 1$ (otherwise $n=1$ or $t=n$ and the statement is trivial), Lemma 4.1 yields

$$
v_{1} \exp \left(-c_{4}(1+\log B)\right)<v_{1}\left(u_{1} v_{1}^{-1}-1\right),
$$

with $c_{4}:=c_{3} h\left(p_{1}\right) \cdots h\left(p_{\ell}\right) h(q)^{2}$, where

$$
c_{3}=c_{3}(\ell+2):=1.4 \cdot 30^{\ell+5}(\ell+2)^{4.5}
$$

is the constant appearing in the conclusion of Matveev's theorem (10) when $\Lambda$ involves $r=\ell+2$ rational numbers $(r=\ell+2$ and $D=1)$. Now we show that

$$
\frac{v_{1}}{v_{j}}<\exp \left(2 j \log t\left(c_{4}(1+\log B)\right)^{j-1}\right) \quad(j=2, \ldots, t) .
$$

We prove this claim by induction. Combining the above inequality (14) with

$$
v_{1}\left(u_{1} v_{1}^{-1}-1\right)=u_{1}-v_{1}<t v_{2}
$$

implied by (13), we get

$$
\frac{v_{1}}{v_{2}}<\exp \left(\log t+c_{4}(1+\log B)\right) \leq \exp \left(2(\log t) c_{4}(1+\log B)\right) .
$$

Let now $i$ be arbitrary with $2 \leq i<t$, and assume by induction that

$$
\frac{v_{1}}{v_{j}}<\exp \left(2 j \log t\left(c_{4}(1+\log B)^{j-1}\right)\right) \quad \text { for all } j=2, \ldots, i \text {. }
$$


Rewrite (11) as

$$
u_{1}-v_{1}-\cdots-v_{i}=v_{i+1}+\cdots+v_{t} .
$$

Observe that by (12) and (16) (used with $j=i$ ), we have

$$
\begin{aligned}
& h\left(1+\frac{v_{2}}{v_{1}}+\cdots+\frac{v_{i}}{v_{1}}\right)=h\left(\frac{q^{\beta_{1}-\beta_{i}}+\cdots+q^{\beta_{i-1}-\beta_{i}}+1}{q^{\beta_{1}-\beta_{i}}}\right)= \\
& =\log \left(q^{\beta_{1}-\beta_{i}}+\cdots+q^{\beta_{i-1}-\beta_{i}}+1\right) \leq \log \left(t q^{\beta_{1}-\beta_{i}}\right)= \\
& =\log t+\log \left(\frac{v_{1}}{v_{i}}\right)<(2 i+1)(\log t)\left(c_{4}(1+\log B)\right)^{i-1} .
\end{aligned}
$$

Hence, Lemma 4.1 yields

$$
\begin{gathered}
v_{1} \exp \left(-(2 i+1)\left(c_{4}(1+\log B)\right)^{i}\right)< \\
<v_{1}\left(u_{1} v_{1}^{-1}\left(1+\frac{v_{2}}{v_{1}}+\cdots+\frac{v_{i}}{v_{1}}\right)^{-1}-1\right)< \\
<v_{1}\left(1+\frac{v_{2}}{v_{1}}+\cdots+\frac{v_{i}}{v_{1}}\right)\left(u_{1} v_{1}^{-1}\left(1+\frac{v_{2}}{v_{1}}+\cdots+\frac{v_{i}}{v_{1}}\right)^{-1}-1\right) .
\end{gathered}
$$

The above inequality together with

$$
\begin{gathered}
v_{1}\left(1+\frac{v_{2}}{v_{1}}+\cdots+\frac{v_{i}}{v_{1}}\right)\left(u_{1} v_{1}^{-1}\left(1+\frac{v_{2}}{v_{1}}+\cdots+\frac{v_{i}}{v_{1}}\right)^{-1}-1\right)= \\
=u_{1}-v_{1}-\cdots-v_{i}<t v_{i+1}
\end{gathered}
$$

obtained from (17), implies the inequality

$$
\begin{aligned}
\frac{v_{1}}{v_{i+1}} & <\exp \left(\log t+(2 i+1)(\log t)\left(c_{4}(1+\log B)\right)^{i}\right) \\
& <\exp \left((2 i+2)(\log t)\left(c_{4}(1+\log B)\right)^{i}\right)
\end{aligned}
$$

which completes the induction step. Hence, our claim (15) follows. Now note that either $B=\beta_{1}$ or $B \in\left\{\alpha_{1}, \ldots, \alpha_{\ell}\right\}$. In the latter case we have $2^{B} \leq n \leq t q^{\beta_{1}}$, so $\beta_{1} \geq c_{5} B-c_{6} \log t$, where $c_{5}:=\log 2 / \log q$ and $c_{6}:=1 / \log q$. Since $q \geq 2$, it follows that the inequality

$$
\beta_{1} \geq c_{5} B-c_{6} \log t
$$

holds both when $B=\beta_{1}$ and when $B \in\left\{\alpha_{1}, \ldots, \alpha_{\ell}\right\}$.

Further, $\log n / \log \left(p_{1} \cdots p_{\ell}\right) \leq B \leq \log n / \log 2$ showing that

$$
\log \log n-c_{7} \leq \log B \leq \log \log n+c_{8},
$$


where $c_{7}:=\log \log \max \left\{3, p_{1} \ldots p_{\ell}\right\}$ and $c_{8}:=-\log \log 2$. Note now that since $q \notin\left\{p_{1}, \ldots, p_{\ell}\right\}$, it follows that $v_{t}=1$. Setting $j=t$ in (15) and taking logarithms we get

$$
c_{5} B-c_{6} \log t \leq \beta_{1} \leq c_{9} t \log t\left(c_{4}(1+\log B)\right)^{t-1},
$$

where we can take $c_{9}:=\max \left\{1,2(\log q)^{-1}\right\}$. If the left-hand side of (19) is smaller than $c_{5} B / 2$, we then get that

$$
\log t>c_{10} B
$$

where $c_{10}:=c_{5} /\left(2 c_{6}\right)$, therefore

$$
t>e^{c_{10} B}>n^{c_{11}},
$$

where $c_{11}:=c_{10} / \log \left(p_{1} \cdots p_{\ell}\right)$, which for large $n$ is better than the inequality we are after. If the left-hand side of (19) is at least $c_{5} B / 2$, then by taking logarithms we get

$$
\log B-c_{12}<(t-1) \log \left(c_{4}(1+\log B)\right)+\log t+\log \log t
$$

where $c_{12}:=-\log \left(c_{5} / 2\right)+\log c_{9}$. From here, we get right-away that in fact

$$
t>(1+o(1)) \frac{\log B}{\log \log B}
$$

as $B \rightarrow \infty$. Combining this with (18), we get that for every $\varepsilon>0$, taking $c_{13}:=1-\varepsilon$, the inequality

$$
t>c_{13} \frac{\log \log n}{\log \log \log n}
$$

holds for all $n>n_{0}(\varepsilon)$, where $n_{0}(\varepsilon)$ is effectively computable in terms of $\varepsilon$ and $p_{1}, \ldots, p_{\ell}, q$. Hence, the statement follows.

\section{Proof of Theorem 2.3}

To prove Theorem 2.3 we use the method of Bertók and Hajdu, described in [3].

Proof of Theorem 2.3. . If $w_{S_{1}}^{+}(n)+w_{S_{2}}^{+}(n)=2$, then it is clear that the only solution is $n=1$, so we suppose that $w_{S_{1}}^{+}(n)+w_{S_{2}}^{+}(n) \geq 3$. We describe our method in detail only in the case when $S_{1}=\{3\}$, $S_{2}=\{2,5\}$. The other cases can be handled similarly. In this case we have five equations to solve, namely:

$$
\begin{aligned}
3^{a_{1}} & =2^{b_{1}} \cdot 5^{c_{1}}+2^{b_{2}} \cdot 5^{c_{2}}, \\
3^{a_{1}} & =2^{b_{1}} \cdot 5^{c_{1}}+2^{b_{2}} \cdot 5^{c_{2}}+2^{b_{3}} \cdot 5^{c_{3}}, \\
3^{a_{1}}+3^{a_{2}} & =2^{b_{1}} \cdot 5^{c_{1}}, \\
3^{a_{1}}+3^{a_{2}} & =2^{b_{1}} \cdot 5^{c_{1}}+2^{b_{2}} \cdot 5^{c_{2}}, \\
3^{a_{1}}+3^{a_{2}}+3^{a_{3}} & =2^{b_{1}} \cdot 5^{c_{1}} .
\end{aligned}
$$


To find all solutions of the above equations we apply the algorithm introduced in [3]. Here we only sketch the method and concentrate on how to use it for our present equations. For the detailed description of the general method, see [3]. First by an exhaustive search we find all 'small' solutions of the equations in non-negative integers $a_{i}, b_{i}, c_{i}$, $(i=1,2,3)$. Then after modifying the equations appropriately we try to find a modulus $m$ such that the modified equation has no solutions modulo $m$. We illustrate the method by solving the equation

$$
3^{a_{1}}=2^{b_{1}} \cdot 5^{c_{1}}+2^{b_{2}} \cdot 5^{c_{2}} .
$$

By an exhaustive search we get that this equation has only five solutions with $a_{1}, b_{1}, b_{2}, c_{1}, c_{2} \leq 100$, namely

$$
\begin{aligned}
\left(a_{1}, b_{1}, b_{2}, c_{1}, c_{2}\right)= & (1,0,0,1,0),(2,0,0,3,0),(4,4,1,0,0), \\
& (2,0,1,2,0),(3,1,0,0,2),
\end{aligned}
$$

yielding $n=3,9,27,81$. Note that $9=3^{2}$ can be represented in two ways, since $9=1+8=5+4$. We suspect that the equation has no other solutions. First it can be seen that if both $b_{1}$ and $b_{2}$ are greater than zero then this equation has no solutions modulo 2 . The same argument applies for $c_{1}, c_{2}$ modulo 5 , thus we conclude that we have to solve the following two equations:

$$
\begin{aligned}
& 3^{a_{1}}=1+2^{b_{2}} \cdot 5^{c_{2}}, \\
& 3^{a_{1}}=5^{c_{1}}+2^{b_{2}} .
\end{aligned}
$$

Since in every 'small' solution the exponent of 3 is at most 4 , then instead of the equations above, we consider

$$
\begin{aligned}
& 3^{5} \cdot 3^{a_{1}^{\prime}}=1+2^{b_{2}} \cdot 5^{c_{2}}, \\
& 3^{5} \cdot 3^{a_{1}^{\prime}}=5^{c_{1}}+2^{b_{2}},
\end{aligned}
$$

respectively, where every exponent is a non-negative integer. If our expectation is true, then these equations have no solutions. To prove this, we show that these equations are already not solvable locally, modulo an appropriately chosen modulus. About how to find such a modulus, we refer once again to [3]. Now we only state that if we choose $m$ to be $3^{5} \cdot 7 \cdot 13 \cdot 17 \cdot 19 \cdot 37 \cdot 73 \cdot 97 \cdot 109 \cdot 163 \cdot 193 \cdot 257 \cdot 433$. $487 \cdot 577 \cdot 769 \cdot 1153 \cdot 1297 \cdot 2593 \cdot 3457 \cdot 10369$, then as one can check, equation (22) has no solutions modulo $m$. Thus, in (20), $a_{1}$ has to be less than or equal to 4 . By checking every possibility we get that this equation has three solutions, namely

$$
\left(a_{1}, b_{1}, b_{2}, c_{1}, c_{2}\right)=(1,0,0,1,0),(2,0,0,3,0),(4,4,1,0,0) .
$$


Similarly, if $m=3^{5} \cdot 7 \cdot 13 \cdot 17 \cdot 19 \cdot 37 \cdot 73 \cdot 97 \cdot 109 \cdot 163 \cdot 193 \cdot 433 \cdot 577 \cdot 769$, then equation (23) has no solutions modulo $m$, thus we only have to check (21) with $a_{1} \leq 4$. In this case we get the remaining two 'small' solutions. The other equations can be handled similarly. Finally, we mention that an appropriately chosen divisor of $M=2^{16} \cdot 3^{10} \cdot 5^{8} \cdot 7$. $13 \cdot 17 \cdot 19 \cdot 163 \cdot 37 \cdot 433 \cdot 193 \cdot 97 \cdot 73 \cdot 257 \cdot 109 \cdot 577 \cdot 769 \cdot 487 \cdot 1153 \cdot 1297$. $1459 \cdot 2593 \cdot 2917 \cdot 3457 \cdot 3889 \cdot 10369 \cdot 1373 \cdot 3137 \cdot 12289 \cdot 17497 \cdot 18433 \cdot$ $39367 \cdot 52489 \cdot 65537 \cdot 50177 \cdot 139969 \cdot 147457 \cdot 209953 \cdot 331777 \cdot 472393$. $114689 \cdot 268913 \cdot 470597 \cdot 629857 \cdot 746497 \cdot 786433 \cdot 839809 \cdot 995329 \cdot 614657$ is sufficient for every equation under investigation. (Certainly, one can take $m=M$ in each case, however, then the computation time would be enormous.)

\section{AcKnowledgements}

The authors are grateful to the referees for their helpful comments.

\section{REFERENCES}

[1] Zs. Ádám, L. Hajdu and F. Luca, Representing primes as linear combinations of pure powers, Acta Arith. 138 (2009), 101-107.

[2] Cs. Bertók, Representing integers as sums or differences of general power products, Acta Math. Hung. 141 (2013), 291-300.

[3] Cs. Bertók and L. Hajdu, A Hasse-type principle for exponential Diophantine equations and its applications, Math. Comp., electronically published on July 16, 2015, DOI: http://dx.doi.org/10.1090/S0025-5718-2014-02815-9 (to appear in print).

[4] Y. Bugeaud, M. Cipu and M. Mignotte, On the representation of Fibonacci and Lucas numbers in an integer base, Ann. Math. Québec 37 (2013), 31-43.

[5] V. S. Dimitrov and E. W. Howe, Lower bounds on the lengths of double-base representations, Proc. Amer. Math. Soc. 139 (2011), 3423-3430.

[6] P. Erdős, C. Mauduit and A. Sárközy, On arithmetic properties of integers with missing digits. I. Distribution in residue classes, J. Number Theory $\mathbf{7 0}$ (1998), 99-120.

[7] P. Erdős, C. Mauduit and A. Sárközy, On arithmetic properties of integers with missing digits. II. Prime factors, Discrete Math. 200 (1999), 149-164. Paul Erdős memorial collection.

[8] J.-H. Evertse, The number of solutions of decomposable form equations, Invent. Math. 122 (1995), no. 3, 559-601.

[9] J. H. Evertse and K. Györy, On the number of solutions of weighted unit equations, Compositio Math. 66 (1988), 329-354.

[10] L. Hajdu and F. Luca, On the length of arithmetic progressions in linear combinations of S-units, Arch. Math. 94 (2010), 357-363. Correction ibid. 103 (2014), 399-400.

[11] L. Hajdu and R. Tijdeman, Representing integers as linear combinations of powers, Publ. Math. Debrecen 79 (2011), 461-468. 
[12] L. Hajdu and R. Tijdeman, Representing integers as linear combinations of power products, Arch. Math. 98 (2012), 527-533.

[13] F. Luca, Distinct digits in base $b$ expansions of linear recurrence sequences, Quaest. Math. 23 (2000), 389-404.

[14] E. M. Matveev, An explicit lower bound for a homogeneous rational linear form in the logarithms of algebraic numbers II, Izv. Ross. Akad. Nauk Ser. Mat. 64 (2000), 125-180; translation in Izv. Math. 64 (2000), 1217-1269.

[15] C. Mauduit, C. Pomerance and A. Sárközy, On the distribution in residue classes of integers with a fixed sum of digits, Ramanujan J. 9 (2005), 45-62.

[16] C. Mauduit and A. Sárközy, On the arithmetic structure of sets characterized by sum of digits properties, J. Number Theory 61 (1996), 25-38.

[17] C. Mauduit and A. Sárközy, On the arithmetic structure of the integers whose sum of digits is fixed, Acta Arith. 81 (1997), 145-173.

[18] M. B. Nathanson, Geometric group theory and arithmetic diameter, Publ. Math. Debrecen 79 (2011), 563-572.

[19] A. Pethö and R. F. Tichy, On digit expansions with respect to linear recurrences, J. Number Theory 33 (1989), 243-256.

[20] H. G. Senge and E. G. Straus, PV-numbers and sets of multiplicity, Period. Math. Hungar. 3 (1973), 93-100.

[21] C. L. Stewart, On the representation of an integer in two different bases, J. Reine Angew. Math. 319 (1980), 63-72.

[22] S. G. Wagner, Numbers with fixed sum of digits in linear recurrent number systems, Ramanujan J. 14 (2007), 43-68.

Cs. Bertók

University of Debrecen, Institute of Mathematics

H-4010 Debrecen, P.O. Box 12.

HUNGARY

L. HAJDU

University of Debrecen, Institute of Mathematics

H-4010 Debrecen, P.O. Box 12.

HUNGARY

F. LUCA

SChool of Mathematics

Private Bag X3, Wits 2050

SOUTh AFrica

D. Sharma

School of Mathematics

Tata Institute of Fundamental Research

Homi Bhabha Road, Mumbai - 400005

INDIA

E-mail address: bertok.csanad@science.unideb.hu

E-mail address: hajdul@science.unideb.hu

E-mail address: florian.luca@wits.ac.za

E-mail address: divyum@math.tifr.res.in 